\title{
Dual-Peak Post-Stroke Edema in a Patient with Infective Endocarditis
}

Peris R. Castaneda, MA ${ }^{1}$; Joseph R. Linzey, MD;

D. Andrew Wilkinson, $\mathrm{MD}^{2}$; Aditya S. Pandey, $\mathrm{MD}^{2}$

\section{ABSTRACT}

\section{Introduction}

Cerebral edema following ischemic stroke due to infective endocarditis classically peaks between 3 and 5 days.

\section{Case Presentation}

The authors describe the case of a 23-year-old man with infective endocarditis complicated by ischemic stroke who developed cerebral edema that peaked at 8 days and 15 days post-stroke and subsequently resolved without neurosurgical intervention. To the best of the authors' knowledge, there is no reported case of dual peaks in edema occurring in a stroke patient with infective endocarditis.

\section{Discussion}

Recognition of dual-peak edema as a phenomenon in infective endocarditis-related stroke is essential to guide neurosurgical intervention and may be important for determining optimal timing of cardiac valve-replacement surgery.

\section{Conclusion}

Further investigations are necessary to understand the frequency of such occurrences in patients with ischemia related to infective endocarditis. pcastane@med.umich.edu; jlinzey@med.umich.edu; dawilki@med.umich.edu; adityap@med.umich.edu

Corresponding Author: Aditya S. Pandey, MD adityap@med.umich.edu, 734-615-4486

Conflicts of Interest: The authors report no conflicts of interest.

Key Words: infective endocarditis; stroke; cerebral edema; blood brain barrier; ischemic stroke

\section{Introduction}

nfective endocarditis (IE) is a rare disease, with a reported annual incidence of 10,000-15,000 cases per year in the United States. ${ }^{1}$

The in-hospital mortality rate for IE has remained stable at 20\% despite improvements in antibiotics and cardiac surgery. ${ }^{1,2}$ An estimated 24-40\% of IE cases are associated with symptomatic neurological complications, with ischemic stroke being the most common neurological complication. In fact, a cerebrovascular event is often the presenting symptom for patients with IE. 3,4 Ischemic insults in IE patients can lead to edema and mass effect, which complicate the decision for valve replacement. ${ }^{4}$ 
Edema in peri-infarct brain parenchyma, no matter the cause of ischemia, begins to develop 24-72 hours following the onset of ischemic symptoms and peaks at 3-5 days. 5,6 This edema can be well visualized with T2 fluid-attenuated inversion recovery (FLAIR) magnetic resonance imaging (MRI). ${ }^{7}$ Cerebral edema that develops after an ischemic stroke due to IE classically follows this pattern of developing 24-72 hours post-stroke and peaking at 3-5 days post-stroke. ${ }^{8}$ A preliminary literature review of cases describing patients with strokes related to endocarditis was conducted. To the best of our knowledge, there has not been a case reported of a patient with IE presenting with 2 peaks of edema, more than 1 week apart and outside of the 3-5 day time period. We report a patient who had an IE-related cerebral infarct, followed by 2 peaks in cerebral edema: 1 peak occurring at 8 days post-stroke, the other occurring at 15 days post-stroke.

\section{Case Presentation History and Examination}

A 23-year-old man presented to an outside hospital with severe left-sided abdominal bloating and left flank pain. He had no significant past medical history and no significant family history of cardiac valve abnormalities. Social history was significant for current tobacco use of 1 pack per day for 5 years. The patient denied alcohol use or intravenous drug use.

His presenting symptoms were preceded by 3 months of generalized fatigue and a $20 \mathrm{lb}$. weight loss. He also experienced night sweats 3 times per week and had 2 episodes of blurry vision that each resolved after 20 seconds. At an outside hospital, a transthoracic echocardiogram (TTE) demonstrated a bicuspid aortic valve with a $1.5 \mathrm{~cm}$ mobile vegetation of the noncoronary cusp. TTE also revealed moderate to severe aortic insufficiency, along with fenestrations of the aortic valve leaflets. Findings were suggestive of bicuspid aortic valve with endocarditis.

A computed tomography (CT) scan of the abdomen and pelvis demonstrated a splenic infarct.
Blood cultures were ordered at the outside hospital, and the patient was started on empiric vancomycin, cefepime, and ciprofloxacin. Blood cultures grew Streptococcus gordonii. The patient was transferred to our hospital and admitted.

\section{Clinical Course}

Hospital Day (HD) 1: On admission, the patient was noted to be hemodynamically stable and neurologically intact. Transesophageal echocardiography (TEE) at our hospital demonstrated vegetation and perforation of a bicuspid aortic valve. He began a 6-week course of IV ceftriaxone. On his first hospital day (HD), he experienced worsening neck pain but had a grossly intact neurological examination. The cardiothoracic surgery service was consulted for possible aortic valve replacement surgery. Valve surgery was planned for HD 5.

HD 2-3 (confirmed infarct): On HD 2, the patient was noted to have slurring of speech that had not been present the previous evening. Neurological exam was significant for left facial droop and left arm drift. A CT scan of the head was significant for non-enhancing hypodensity in the right insular cortex concerning for possible embolic infarct (Figure 1A). MRI of the brain on HD 3 demonstrated cerebral edema surrounding the previously infarcted brain parenchyma (Figure 1B). The patient was not a tissue plasminogen activator (TPA) candidate, as he was outside of the standard TPA window with unknown time since last normal. No acute neurosurgery interventions were performed. Due to the stroke, valve surgery on HD 5 was deemed to carry too great a risk for hemorrhagic conversion of infarct during surgery. Cardiothoracic surgery service recommended that surgery be postponed for at least 1 week to allow the infarct to mature. Of note, computed tomography angiography (CTA) of the head and neck on HD 3 demonstrated a $2 \mathrm{~mm}$ aneurysm at the bifurcation of the right middle cerebral artery (MCA) at the second marginal coronary artery (M2); repeat CTA was planned for 
1 day prior to anticipated cardiac surgery to assess the stability of the aneurysm.

HD 3-HD 10 (post-stroke days 1-8): The patient's neurological exam continued to improve between
HD 3 and HD 6 and remained stable on HD 7 to HD 9. On HD 10 (8 days after infarct), a progress report noted that the patient had had no acute events the previous day or overnight, and his neurological exam that morning was stable from the previous

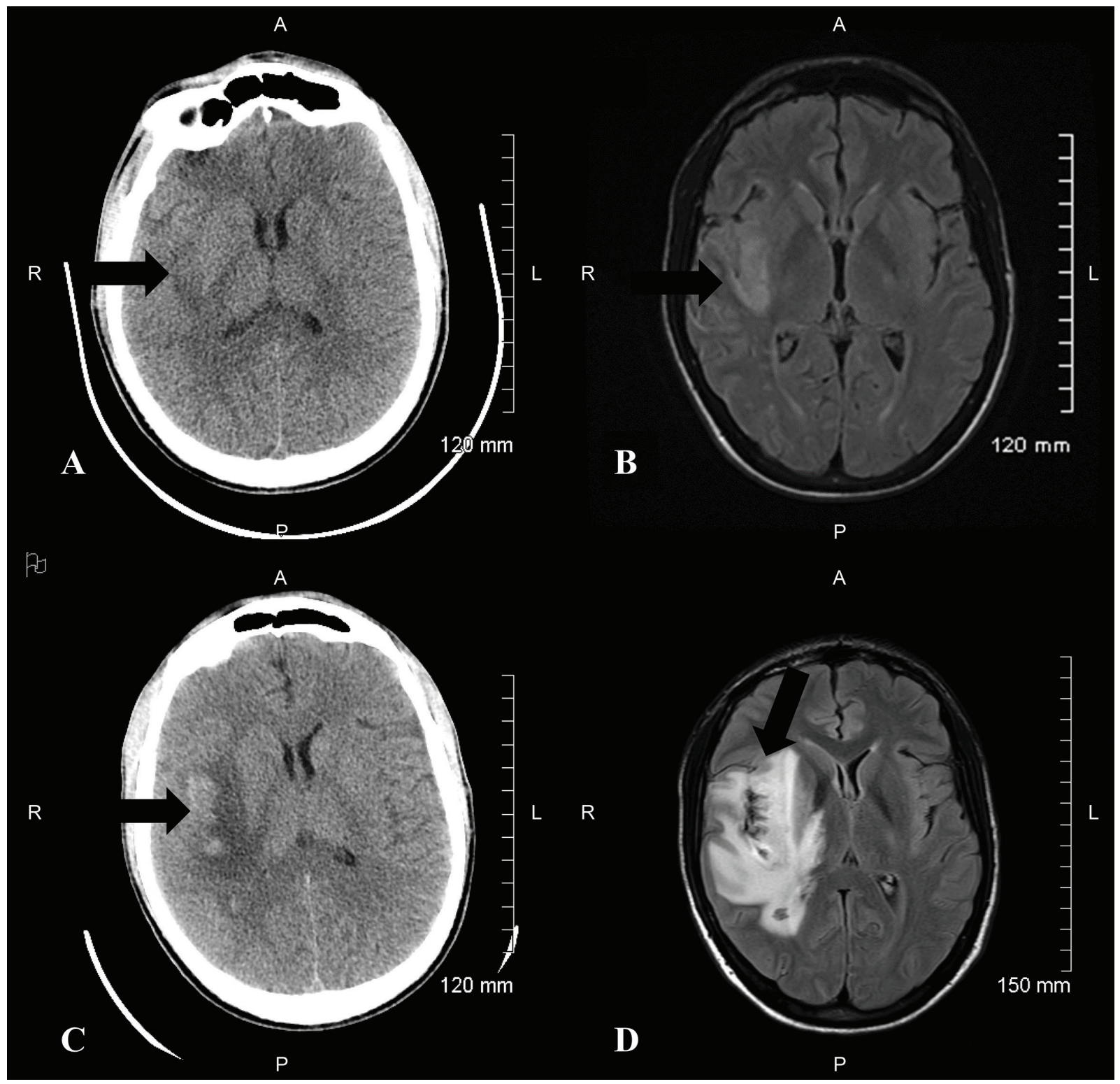

FIGURE 1. (A) Presenting computed tomography (CT) scan of the head on HD 2 (post-stroke day 0). Significant for a non-enhancing hypodensity in the right insular cortex, concerning for a possible embolic infarct (arrow). (B) T2 fluid-attenuated inversion recovery (FLAIR) magnetic resonance image (MRI) from HD 3 (post-stroke day 1) demonstrating hyperintensity consistent with cerebral edema following an acute ischemic stroke (arrow). (C) Head CT on HD 10 (post-stroke day 8) demonstrated interval progression of the evolving infarct with increased petechial hemorrhages. Increased cerebral edema is also present surrounding infarcted region of brain parenchyma (arrow). (D) T2 FLAIR MRI on HD 18 (post-stroke day 16) demonstrates significant progression of the cerebral edema with mass effect upon the right lateral ventricle and $6 \mathrm{~mm}$ of midline shift to the left. There is no significant change in the extent of ischemia or hemorrhage. 
day. Scheduled pre-operative CTA of the head on HD 10 (1 day prior to anticipated valve surgery) revealed evolving infarct of the insular cortex within the area of the right MCA, with increasing petechial hemorrhages along the superior and inferior area of the infarct. Increased cerebral edema surrounding the area of infarcted brain parenchyma was also present (Figure 1C). The patient remained neurologically stable. Secondary to CTA findings, cardiothoracic surgery deferred the patient's surgery for at least 1 more week with plan for repeat CTA prior to surgery.

HD 17-HD 18 (post-stroke days 15-16): Routine preoperative CTA on HD 17 (15 days after infarct) was concerning for interval progression of edema in the right cerebral hemisphere and mass effect with midline shift. A follow-up MRI on HD 18 (16 days after infarct) demonstrated significant progression of cerebral edema with new mass effect upon the right lateral ventricle with $6 \mathrm{~mm}$ of right-to-left midline shift (Figure 1D).

The neurology service was consulted for interpretation of the CTA findings. Their assessment of the changes included possible further progression of the patient's original infarct versus overlying cerebritis due to infection from septic emboli. The patient was transferred to the cardiac care unit (CCU) for closer monitoring and frequent neurological checks.

\section{CCU and Outcome}

During his stay in the CCU, the patient did not experience changes in his neurological symptoms. He had neurological checks every hour on his first CCU day, which were de-escalated to every 3 hours on his third CCU day (HD 19). Cardiothoracic surgery and neurosurgery consults agreed that, due to the patient's risk for heart failure and further embolic events, the patient should not be discharged until a valve replacement could be performed. A brain MRI on HD 25 (23 days after infarct) revealed a reduction in pathologic contrast enhancement in the right cerebral hemispheric lesion, consistent with improving brain injury from septic emboli and cerebritis (Figure 2A). A repeat MRI on HD 33 (31 days after infarct) appeared stable with decreasing T2/FLAIR signal and no novel areas of diffusion restriction (Figure 2B).

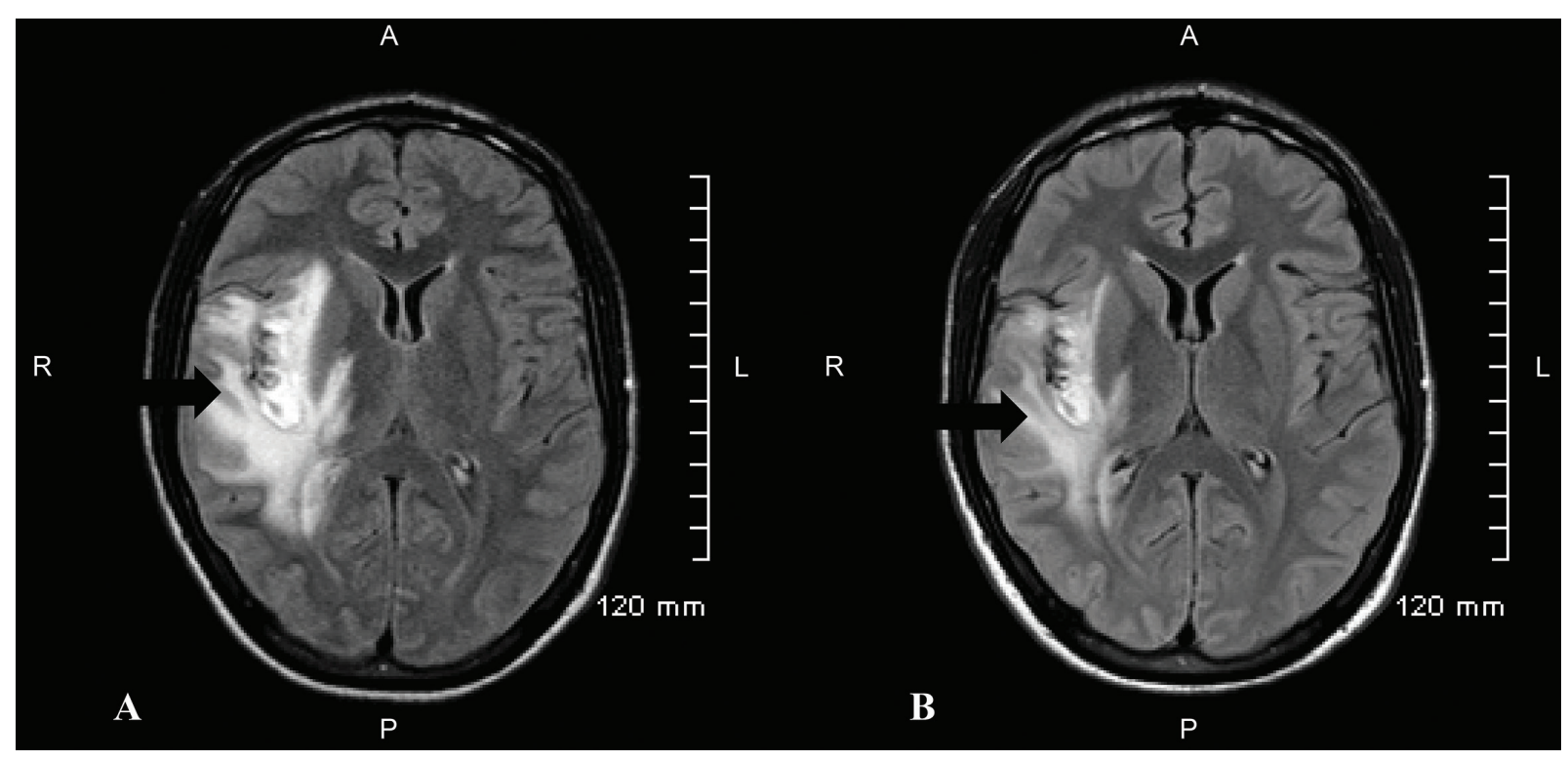

FIGURE 2. (A) T2/FLAIR MRI from HD 25 (post-stroke day 23) demonstrating reduction in pathologic contrast enhancement in the right cerebral lesion. (B) T2/FLAIR MRI from HD 32 (post-stroke day 30) showing mild interval decrease in the degree of parenchymal edema. 
The patient received a bioprosthetic aortic valve replacement 38 days after confirmation of stroke. The native valve was cultured and found to have no growth. His postoperative course was complicated by respiratory insufficiency, hypotension, and cardiac dysfunction requiring inotropic support. He was discharged 6 days after surgery. A follow-up visit 4 months after surgery revealed that the patient was back to participating in all of his normal activities.

\section{Discussion}

Edema in the patient presented here continued to progress beyond the 3-5 day peak that is well documented in the literature. 5,6,7 Repeat head CT and MRI scans revealed increasing cerebral edema at 8 and 15 days following infarct. There are several potential explanations for this occurrence.

Cerebral edema, or excess fluid in the intracellular or extracellular spaces of the brain, is commonly observed in a variety of brain injuries, including ischemic stroke. Two types of cerebral edema are of particular importance in ischemic stroke: cytotoxic edema and vasogenic edema. ${ }^{9}$ Vasogenic edema results from the disruption of the blood brain barrier (BBB). Breakdown of the BBB following brain injury allows normally excluded plasma constituents to enter the cerebral parenchyma, osmotically drawing fluid into the parenchyma. In cytotoxic edema, the BBB remains intact; however, cellular elements swell as the result of failing ATP-dependent ion transport and the subsequent retention of intracellular sodium and water. ${ }^{9,10} \mathrm{Fol}-$ lowing ischemic stroke, cerebral edema is initially cytotoxic and later vasogenic. ${ }^{11}$

One explanation for the increase in edema observed on this patient's imaging at days 8 and 15 may be the disruption of the BBB in the context of infective endocarditis leading to cerebritis. A ruptured BBB allows large molecules to enter the brain parenchyma, resulting in vasogenic edema; thus, infectious material present within the vasculature may be able to leak into the parenchyma. ${ }^{12,13}$ In a patient with infective endocarditis, breakdown of the BBB may enable the entry of the infective agent or antigenic material into the brain parenchyma. Additionally, systemic inflammation, such as that caused by infective endocarditis, has been shown to exacerbate BBB disruption in mice. ${ }^{14}$ It is possible that an atypically large BBB disruption in this patient and subsequent infection of the brain parenchyma with known hematogenous Streptococcus gordonii resulted in vasogenic edema with an uncommon pattern.

Alternatively, increasing vasogenic edema has been reported in the late subacute stage. 15,16 A study of one animal model has described a biphasic opening of the BBB after cerebral artery occlusion. The first BBB opening occurred shortly after recirculation, whereas the second occurred 5-72 hours after recirculation. ${ }^{17}$ It is possible that the patient's progression of edema followed an extended version of this biphasic pattern representing a clinically relevant model of this laboratory phenomenon; nonetheless, edema in the same location 15 days post-stroke is unlikely explained by this phenomenon.

The development of cerebral abscesses from cerebritis provides yet another possible explanation. Hematogenous abscesses can develop in the brain secondary to endocarditis. Abscesses form in response to pyogenic bacterial infection of the parenchyma and begin as localized areas of cerebritis before developing into enclosed pyemic vesicles. ${ }^{18}$ The development of brain abscesses can be presented in four stages: early cerebritis occurring at 1-4 days; late cerebritis occurring at 4-10 days; early capsule formation occurring at 11-14 days; and late capsule formation occurring at 14 days and beyond. ${ }^{19}$ This timing pattern seems in accordance with some of the patient's imaging results: peak cerebritis at 8 days and further progression of edema concerning for cerebritis at 15 days. However, hematogenous abscesses such as those seen in cases of endocarditis are usually multiple. ${ }^{18}$ Accordingly, the cerebral edema visualized on days 8 and 15 post-stroke are unlikely explained by developing cerebral abscesses. 
Our report is limited by the absence of imaging between post-stroke days 1 and 8 and between post-stroke days 8 and 15. As a result, we are unable to track the precise course of the development of edema. Indeed, when we discuss the phenomenon of dual peaks in edema, we refer to 2 observable increases in edema on post-stroke days 8 and 15 when imaging was performed, recognizing that the true peaks may have occurred on different days. Because the patient's neurological function did not deteriorate and, in fact, improved moderately from the time of stroke, it is likely that the subsequent increases in edema do not represent the sequelae of a re-stroke but rather are the result of a single stroke on HD 2.

\section{Conclusion}

Much of the literature describing post-stroke vasogenic edema does not specifically address ischemic events caused by IE. Because the emboli causing stroke are infective in cases of $\mathrm{IE}$, there are special factors to consider. It is possible that the initial loss of the BBB in this patient allowed infective thrombotic residue to leak into the edematous area and cause subsequent cerebritis that propagated progressive cerebral edema 15 days or more post-stroke. Further analysis into the prevalence and natural history of this phenomenon is needed to guide the clinical management of patients presenting with ischemic stroke secondary to IE. For instance, this atypical time course of edema may have implications for the timing of valve surgery, as occurred with our patient. It is also important to recognize that a second peak of edema may reflect cerebritis which resolves on its own. Recognition of this phenomenon is essential to avoid potentially unnecessary neurosurgical intervention when the second peak of edema arrives.

\section{References}

1. Slipczuk L, Codolosa JN, Davila CD, et al. Infective endocarditis epidemiology over five decades: a systematic review. PLoS One. 2013;8(12):e82665. doi:10.1371/journal.pone.0082665
2. Goulenok T, Klein I, Mazighi M, et al. Infective endocarditis with symptomatic cerebral complications: contribution of cerebral magnetic resonance imaging. Cerebrovasc Dis. 2013;35(4):327-336. doi:10 .1159/000348317

3. Hess A, Klein I, lung B, et al. Brain MRI findings in neurologically asymptomatic patients with infective endocarditis. Am J Neuroradiol. 2013;34(8):15791584. doi:10.3174/ajnr.A3582

4. Morris NA, Matiello M, Lyons JL, Samuels MA. Neurologic complications in infective endocarditis: identification, management, and impact on cardiac surgery. Neurohospitalist. 2014;4(4):213-222. doi:10.1177/1941874414537077

5. Dostovic Z, Dostovic E, Smajlovic D, Avdic O. Brain edema after ischaemic stroke. Med Arch. 2016;70(5):339-341. doi:10.5455/medarh.2016.70 .339-341

6. Hacke W, Schwab S, Horn M, Spranger M, De Georgia $\mathrm{M}$; von Kummer R. "Malignant" middle cerebral artery territory infarction: clinical course and prognostic signs. Arch Neurol. 1996;53(4):309-315. doi:10.1001/archneur.1996.00550040037012

7. Colen TW, Gunn M, Cook E, Dubinsky T. Radiological manifestations of extracardiac complications of infective endocarditis. Eur Radiol. 2008;18(11):24332445. doi:10.1007/s00330-008-1037-3

8 Leary MC, Caplan LR. Cardioembolic stroke: an update on etiology, diagnosis and management. Ann Indian Acad Neur. 2008;11(5):52-63.

9. Donkin JJ, Vink R. Mechanisms of cerebral edema in traumatic brain injury: therapeutic developments. Curr Opin Neurol. 2010;23(3):293-299. doi:10.1097/ WCO.0b013e328337f451

10. Raslan A, Bhardwaj A. Medical management of cerebral edema. Neurosurg Focus. 2007;22(5):E12.

11. Krieger DW, Demchuk AM, Kasner SE, Jauss M, Hantson L. Early clinical and radiological predictors of fatal brain swelling in ischemic stroke. Stroke. 1999;30:287-292. doi:10.1161/01.STR.30.2.287

12. Abbott NJ, Patabendige AA, Dolman DE, et al. Structure and function of the blood-brain barrier. Neurobiol Dis. 2010;37(1):13-25. doi:10.1016/j.nbd.2009 .07 .030

13. Sandoval KE, Witt KA. Blood-brain barrier tight junction permeability and ischemic stroke. Neurobiol Dis. 2008;32(2):200-219. doi:10.1016/j.nbd.2008 .08 .005

14. Denes A, Ferenczi S, Kovacs KJ. Systemic inflammatory challenges compromise survival after 
experimental stroke via augmenting brain inflammation, blood-brain barrier damage and brain oedema independently of infarct size. J Neuroinflammation. 2011;8:164. doi:10.1186/1742-2094-8-164

15. Durukan A, Tatlisumak T. Acute ischemic stroke: overview of major experimental rodent models, pathophysiology, and therapy of focal cerebral ischemia. Pharmacol Biochem Behav. 2007;87(1):179197. doi:10.1016/j.pbb.2007.04.015

16. Kanekar SG, Zacharia T, Roller R. Imaging of stroke: part 2, pathophysiology at the molecular and cellular levels and corresponding imaging changes.
Am J Roentgenol. 2012;198(1):63-74. doi:10.2214/ AJR.10.7312

17. Kuroiwa T, Ting P, Martinez H, Klatzo I. The biphasic opening of the blood-brain barrier to proteins following temporary middle cerebral artery occlusion. Acta Neuropathol. 1985;68(2):122-129.

18. Muzumdar D, Jhawar S, Goel A. Brain abscess: an overview. Int J Surg. 2011;9(2):136-144. doi:10 .1016/j.ijsu.2010.11.005

19. Erdogan E, Cansever T. Pyogenic brain abscess. Neurosurg Focus. 2008;24(6):E2. doi:10.3171/FOC/ 2008/24/6/E2 